\title{
On Local Nonreflecting Boundary Conditions for Time Dependent Wave Propagation
}

\author{
Marcus J. GROTE* Imbo SIM* \\ (Dedicated to Professor Andrew Majda on the Occasion of his 60th Birthday)
}

\begin{abstract}
The simulation of wave phenomena in unbounded domains generally requires an artificial boundary to truncate the unbounded exterior and limit the computation to a finite region. At the artificial boundary a boundary condition is then needed, which allows the propagating waves to exit the computational domain without spurious reflection. In 1977, Engquist and Majda proposed the first hierarchy of absorbing boundary conditions, which allows a systematic reduction of spurious reflection without moving the artificial boundary farther away from the scatterer. Their pioneering work, which initiated an entire research area, is reviewed here from a modern perspective. Recent developments such as high-order local conditions and their extension to multiple scattering are also presented. Finally, the accuracy of high-order local conditions is demonstrated through numerical experiments.
\end{abstract}

Keywords Absorbing boundary conditions, Scattering problems, Wave equation

2000 MR Subject Classification 65M99

\section{Introduction}

Unbounded domains are often encountered in scientific and engineering applications. Examples are radar and sonar technology, wireless communication, and seismic imaging. Typically the phenomenon of interest is local but embedded in a vast surrounding medium. Although the exterior region may not be truly unbounded, the boundary effects are often negligible, so that one further simplifies the problem by replacing the vast exterior by an infinite medium.

Mathematical models of natural phenomena usually consist of partial differential equations, whose derivation is based on physical conservation laws. Many standard numerical methods, such as finite differences and finite elements, can approximately solve partial differential equations. In fact, they can even handle complicated geometries, inhomogeneous media, and nonlinearity. However, they typically require an artificial boundary, which truncates the unbounded exterior domain, to fit the infinite region on a finite computer. This immediately raises the question:

Which boundary condition guarantees that the solution to the initial-boundary value problem inside the artificial boundary coincides with the solution of the original problem in the unbounded region?

\footnotetext{
Manuscript received June 8, 2009. Published online August 25, 2009.

*Department of Mathematics, University of Basel, Rheinsprung 21, CH-4051 Basel, Switzerland.

E-mail: Marcus.Grote@unibas.ch Imbo.Sim@unibas.ch
} 
If we exhibit a boundary condition, such that the fictitious boundary appears perfectly transparent, we shall call it "exact". Otherwise it will correspond to an approximate boundary condition (also called radiating, absorbing, silent, transmitting, transparent, open, free-space, and one-way boundary conditions, see [1]) and generate some spurious reflection, which travels back and perturbs the solution everywhere in the computational domain. The resulting error in the computer simulation then consists of two independent error components: the discretization error of the numerical method used in the interior and the spurious reflection generated at the fictitious boundary. Unless both error components are reduced systematically, the numerical solution will not converge to the solution of the original problem in the unbounded region.

In this article, we shall restrict ourselves to time dependent scattering problems. Typically a scattering problem consists of an obstacle, a source term $f$, and possibly an incident wave $u^{i}$ (see Figure 1). Scattering problems are common in acoustic, electromagnetic, and elastic wave propagation. Our goal is to calculate numerically the time-dependent wave field $u^{s}$ scattered from the complex, possibly nonlinear, but bounded scattering region.

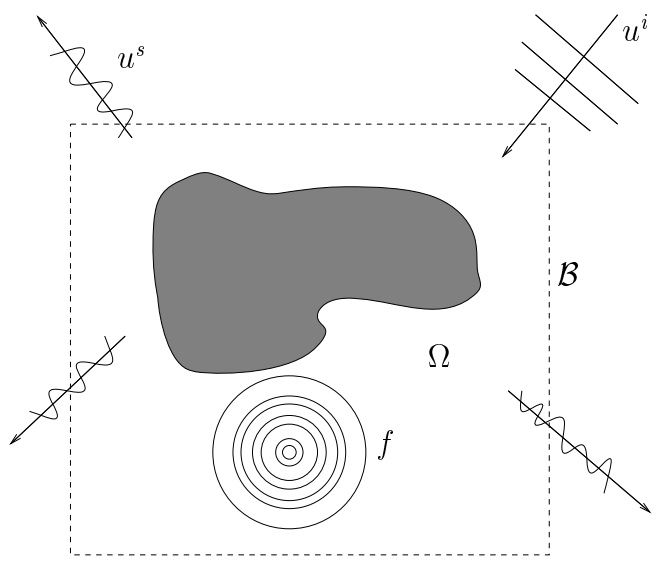

Figure 1 A typical scattering problem consists of an obstacle, a source term $f$, and incoming wave $u^{i}$, and a scattered wave $u^{s}$. The artificial boundary $B$ defines the outer boundary of the computational domain $\Omega$.

In 1974, Smith [2] suggested perhaps the first exact method to restrict the computation to a finite region. Let the computational domain $\Omega$ be bounded by a convex boundary of $n$ line segments (or planar facets in $\mathbb{R}^{3}$ ). Then the restriction to $\Omega$ of the solution in unbounded space consists of a linear combination of $2^{n}$ solutions which satisfy all possible combinations of Dirichlet or Neumann boundary conditions. Unfortunately, this approach has but little practical value, since a rectangular domain requires $2^{6}=64$ independent numerical solutions. This example illustrates a key aspect in the design of improved absorbing boundary conditions: it is not sufficient to construct a new boundary condition; in addition, the computational effort involved must be comparable to that of the numerical method used in the interior. Otherwise it will quickly be dismissed as prohibitively expensive and impractical.

In 1977, Engquist and Majda [3,4] proposed the first hierarchy of absorbing boundary conditions, which allows a systematic reduction of spurious reflection while keeping the artificial boundary at a fixed distance from the scatterer. Their pioneering work, still very much in use 
even today, initiated an entire research area that led to a wide variety of different approaches, such as perfectly matched layers (PML, see [5]), fast integral based formulations (see [6]), semilocal formulations (see [7,8]) and high-order local conditions (see [9-11]) — see [12-14] for review articles and additional references. All these approaches lead to convergent numerical schemes while treating the open boundary at a computational cost comparable to that in the interior.

In this article we shall focus on local absorbing (or nonreflecting) boundary conditions, which are completely local both in space and time. First in Section 2, we introduce the fundamental ideas underlying the derivation of nonreflecting boundary conditions by considering the simple one-dimensional case. Next, we review the original Engquist-Majda conditions [3] for wave propagation in more than one space dimensions, where we exhibit the trade-off between exactness and locality. We also present recent developments of high-order local boundary conditions without high-order derivatives, both for acoustic and electromagnetic waves. Next, in Section 3, we consider the extension of local NBC to multiple scattering, first in one and then in three space dimensions. Finally, in Section 4, we demonstrate the accuracy high-order local conditions via numerical experiments.

\section{Absorbing Boundary Conditions}

To illustrate the fundamental ideas underlying the derivation of absorbing boundary conditions, we begin with a simple one-dimensional problem. In this special situation many basic notions, in particular the exact boundary condition, appear in a very simple form. Nonetheless, we hasten to point out that its appealing simplicity is also misleading: the real challenges in deriving effective absorbing boundary conditions appear only in higher dimensions. Indeed a one-dimensional wave can only propagate in two directions, to the left or to the right. In two or more dimensions, however, waves propagate in infinitely many directions.

\subsection{The one-dimensional wave equation}

Consider the one-dimensional wave equation on the positive real axis,

$$
\frac{\partial^{2} u}{\partial t^{2}}-\frac{\partial^{2} u}{\partial x^{2}}=f, \quad x>0, t>0 .
$$

At the left boundary $x=0$, we require that the solution satisfies

$$
u(0, t)=0, \quad t>0 .
$$

Thus, $u(x, t)$ describes the position of an infinitely (or just very) long vibrating string, attached at its left end; hence, $u=0$ corresponds to the state at rest. The one-dimensional wave equation (2.1) describes the propagation of small perturbations induced by the applied forcing $f(x, t)$. Here we have normalized the propagation speed to one by rescaling time appropriately. The initial conditions of the vibrating string are defined by its position and velocity at $t=0$ :

$$
u(x, 0)=U_{0}(x), \quad \frac{\partial}{\partial t} u(x, 0)=V_{0}(x), \quad x>0 .
$$

It can be shown that the initial-boundary value problem $(2.1)-(2.3)$ is well-posed: it has a unique solution, which depends continuously on $U_{0}, V_{0}$ and $f$. 
We now make the following assumption, which defines the local character of the problem: let the forcing vanish outside a bounded region next to the left boundary, that is, let $f(x, t)=0$ for $x \geq L$ and for all time $t>0$. Then the positive real line separates into two distinct regions: the bounded interval $\Omega=[0, L]$ and the interval $[L, \infty)$, unbounded yet where the forcing vanishes identically. Both regions meet at the artificial boundary $\{x=L\}$, which consists only of a single point. Furthermore, we assume that the string is at rest in the exterior at $t=0: U_{0}(x)=0$ and $V_{0}(x)=0$ for $x \geq L$.

We now wish to simulate numerically the time dependent behavior of the vibrating string in the computational domain $\Omega$. Unfortunately, we cannot apply our favorite numerical scheme in $\Omega$ and simply ignore the new artificial boundary point. On the contrary, we must pay close attention to the new boundary point at $x=L$. Without a boundary condition at $x=L$, the initial value problem (2.1)-(2.3) restricted to $\Omega$ is not even well-posed. To derive a boundary condition, we first need to better understand its role at the artificial boundary. Suppose that a wave propagates to the right inside $\Omega$ and reaches the right boundary at $x=L$. It must not be reflected, for any spurious reflection will travel back into the computational domain and spoil the solution everywhere. This spurious reflection, caused by an inaccurate treatment of the artificial boundary, is not due to finite precision, unlike discretization errors present in any computation. If we find a boundary condition, which lets the waves hit the boundary without any reflection, the solution inside $\Omega$, with that boundary condition imposed at $x=L$, coincides with the restriction to $\Omega$ of the solution in the unbounded region. Hence such a boundary condition is exact.

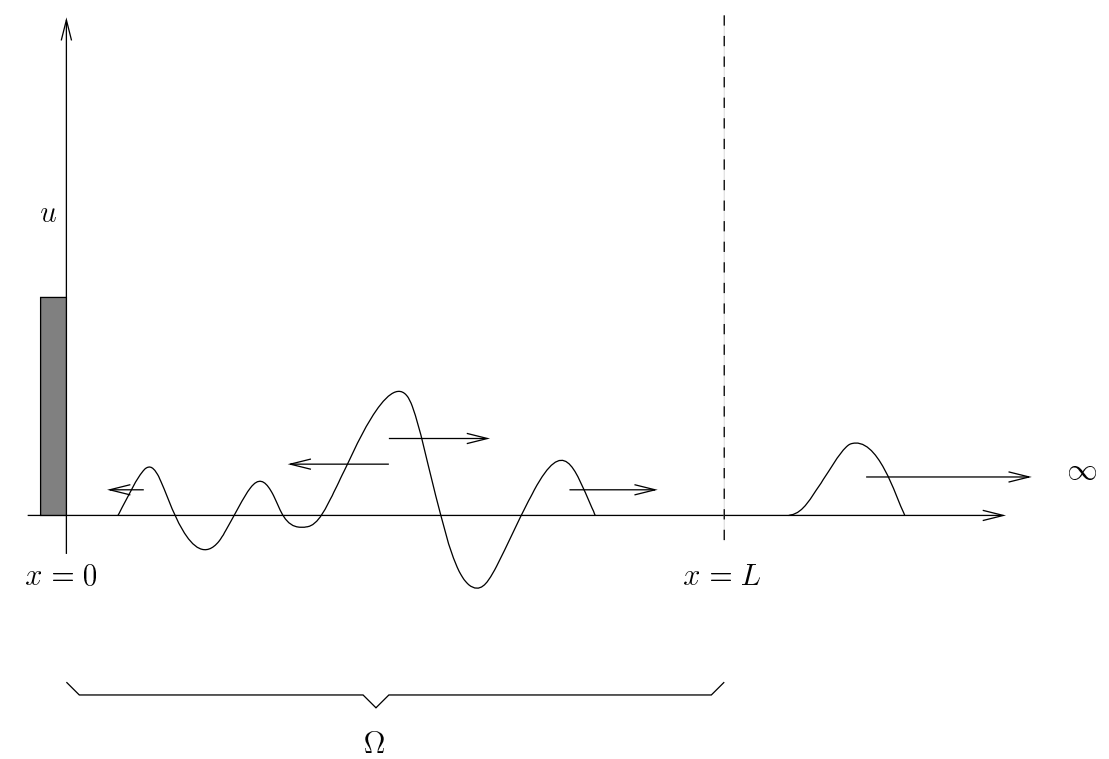

Figure 2 The one-dimensional wave equation: inside the computational domain $\Omega=[0, L]$, the problem can be arbitrarily complicated, but in the exterior region $x \geq L$, we assume that $f(x, t)=0$ for $t>0$ and that $u$ and $\partial_{t} u$ vanish at $t=0$.

Inside the computational domain $\Omega$ waves propagate both to the left and to the right. In the exterior region, however, the absence of any forcing and the zero initial conditions preclude 
the appearance of any waves traveling to the left: there all waves propagate eastward towards infinity (see Figure 2). To derive the exact boundary condition at $x=L$, we first need to separate the incoming from the outgoing waves. To do so, we let $v$ and $w$ be defined by

$$
v=\frac{\partial u}{\partial t}+\frac{\partial u}{\partial x}, \quad w=\frac{\partial u}{\partial t}-\frac{\partial u}{\partial x} .
$$

Since $u$ satisfies the wave equation (2.1) in $x \geq L$, we conclude that

$$
\frac{\partial v}{\partial t}-\frac{\partial v}{\partial x}=0, \quad \frac{\partial w}{\partial t}+\frac{\partial w}{\partial x}=0 .
$$

Thus we can rewrite (2.1) as the first-order hyperbolic system

$$
\frac{\partial}{\partial t}\left[\begin{array}{c}
v \\
w
\end{array}\right]+\left[\begin{array}{cc}
-1 & 0 \\
0 & 1
\end{array}\right] \frac{\partial}{\partial x}\left[\begin{array}{c}
v \\
w
\end{array}\right]=0
$$

Its general solution is

$$
v(x, t)=\phi(x+t) \quad \text { and } \quad w(x, t)=\psi(x-t),
$$

where $\phi$ and $\psi$ are arbitrary functions, which are determined by initial and boundary conditions. Therefore, $v$ is constant on the characteristics $x+t=c$, whereas $w$ remains constant on the characteristics $x-t=c$. Thus $v$ corresponds to incoming waves, whereas $w$ corresponds to outgoing waves. Since there are no incoming waves in $x \geq L$, we have

$$
v(L, t)=0, \quad t>0 .
$$

By applying the definition (2.4) of $v$ in (2.6), we thus obtain the exact nonreflecting boundary condition for the displacement $u(x, t)$,

$$
\left(\frac{\partial}{\partial t}+\frac{\partial}{\partial x}\right) u=0, \quad x=L, t>0 .
$$

Note that the problem inside $\Omega$ can be arbitrarily complicated, since the derivation of the (exact) nonreflecting boundary condition (2.7) depends only on properties in the exterior region.

\subsection{Absorbing boundary conditions in higher dimensions}

We consider a highly complex but local scatterer in unbounded two space dimensions. Although we shall restrict ourselves to the two-dimensional case, much of the present discussion carries over immediately to the three-dimensional case. Thus we consider the wave equation on the two-dimensional infinite plane,

$$
\frac{\partial^{2} u}{\partial t^{2}}-\frac{\partial^{2} u}{\partial x^{2}}-\frac{\partial^{2} u}{\partial y^{2}}=f, \quad t>0,
$$

with the initial conditions

$$
u(x, y, 0)=U_{0}(x, y), \quad \frac{\partial}{\partial t} u(x, y, 0)=U_{1}(x, y), \quad t=0 .
$$

By scaling time appropriately we have normalized the speed of propagation to one. Again the phenomenon of interest is very complicated, possibly nonlinear, but local. Next, we truncate 
the unbounded exterior by an artificial boundary and restrict the computation to the square $\Omega=[-L, L] \times[-L, L]$ (see Figure 1). Outside $\Omega$ we assume that neither source terms nor initial perturbations are present:

$$
U_{0}(x, y)=U_{1}(x, y)=0, \quad f(x, y, t)=0, \quad t>0,(x, y) \in \mathbb{R}^{2} \backslash \Omega .
$$

Again we seek a boundary condition at $(x, y) \in B$, which ensures that all waves reach the exterior region unharmed without generating any unphysical reflection at the fictitious interface. Because of symmetry we only need to consider a single edge of the rectangle, here the right edge at $x=L$. Hence the exterior region lies to the right and the computational domain $\Omega$ to the left of the artificial boundary $\left\{(x, y) \in \mathbb{R}^{2} \mid x=L\right\}$. Since the initial conditions and the forcing vanish identically in the exterior, all waves in the region $x \geq L$ are purely outgoing and must propagate eastward. To avoid any spurious reflection at $x=L$, the exact boundary condition must annihilate all incoming waves. In the previous section we easily derived such an exact nonreflecting boundary condition for the one-dimensional wave equation. Unfortunately, the same approach does not apply in two dimensions. In contrast to the one-dimensional case, any fixed location $(L, y)$ at the artificial boundary receives incoming waves from not one but infinitely many angles of incidence, which propagate in infinitely many directions. The distinction between incoming and outgoing waves is now "infinitely more complicated".

Let $\widehat{u}(x, \xi, \omega)$ denote the Fourier transform of the solution $u(x, y, t)$ in time and in the tangential plane, parallel to the artificial boundary,

$$
\widehat{u}(x, \xi, \omega)=\int_{-\infty}^{\infty} \int_{-\infty}^{\infty} u(x, y, t) \mathrm{e}^{\mathrm{i}(\omega t+\xi y)} \mathrm{d} y \mathrm{~d} t .
$$

Here we have set the solution $u(x, y, t)$ to zero for all previous time $t<0$. Then $u$ is related to $\widehat{u}$ via the inverse Fourier transform, which resembles (2.9) after exchanging $u$ and $\widehat{u}$. Since $u$ satisfies the wave equation (2.8) with $f=0$ for $x \geq L$, its Fourier transform satisfies

$$
\frac{\partial^{2}}{\partial x^{2}} \widehat{u}=\left(\xi^{2}-\omega^{2}\right) \widehat{u}, \quad x \geq L .
$$

To derive an exact nonreflecting boundary condition at $x=L$, we need to relate the normal derivative (here $\partial_{x} u$ ) to tangential and time derivatives (here $\partial_{y} u$ and $\partial_{t} u$ ). From (2.10) we conclude that $\partial_{x} \widehat{u}$ is determined by $\pm \sqrt{\xi^{2}-\omega^{2}} \widehat{u}$. The sign in front of the square root discriminates precisely incoming from outgoing waves; here the correct choice leads to the following exact boundary condition:

$$
\frac{\partial}{\partial x} \widehat{u}=-\mathrm{i} \omega \sqrt{1-\left(\frac{\xi}{\omega}\right)^{2}} \widehat{u}, \quad x=L .
$$

Although this boundary condition ensures the absolute transparency of the artificial boundary, this formulation has but little value in practice. Indeed, we do not seek a boundary condition for $\widehat{u}$ but instead for $u$. In theory we can always compute the inverse transform and thus determine $\partial_{x} u$. However, unlike a polynomial expression, whose inverse Fourier transform yields a local differential operator, the inverse transform of the above expression does not result 
in a simple differential operator because of the square root. Instead, we obtain a so-called pseudo-differential operator, which cannot be evaluated without forward and inverse Fourier transform. As a consequence, the normal derivative $\partial_{x} u$ at any given point on the boundary $(L, y)$ depends on past values of $u$ on the entire line $x=L$, and cannot be computed locally either in space or time.

"... unfortunately, these (perfectly absorbing) boundary conditions have to be nonlocal in both space and time", - Engquist and Majda, 1977.

To overcome the difficulties associated with the nonlocal nature of the exact boundary condition (2.11), we can replace the above pseudo-differential operator by an approximate differential operator. In doing so we give up on the absolute transparency of the artificial boundary and accept some spurious reflection. Such absorbing boundary conditions were proposed by Engquist and Majda [3] in 1977, and we now briefly recall the fundamental ideas underlying this popular approach.

The Fourier transform of a differential operator always results in a polynomial expression in the frequency domain. For instance, the Fourier transform of the differential operator $\partial_{y y}$ yields the polynomial $-\xi^{2}$. Conversely every polynomial in Fourier space corresponds to a (local) differential operator in physical space. Thus, the inverse Fourier transform of a polynomial in $s=\frac{\xi}{\omega}$, which approximates $\sqrt{1-s^{2}}$, will yield a differential operator, which can be used as an (approximate) absorbing boundary condition in physical space.

For $s$ sufficiently small, we approximate $\sqrt{1-s^{2}}$ by the first few terms of its Taylor expansion:

$$
\sqrt{1-s^{2}}=1-\frac{s^{2}}{2}+O\left(s^{4}\right), \quad|s| \rightarrow 0 .
$$

We now replace the square root in (2.11) by the leading term in the Taylor expansion, that is $\sqrt{1-s^{2}} \simeq 1$, and perform the inverse Fourier transform to obtain

$$
\frac{\partial \widehat{u}}{\partial x} \simeq-\mathrm{i} \omega \widehat{u} \Rightarrow\left(\frac{\partial}{\partial t}+\frac{\partial}{\partial x}\right) u=0, \quad x=L .
$$

This is the first-order Engquist-Majda boundary condition, which contains only first derivatives of the solution. It coincides with the exact boundary condition (2.7) for the one-dimensional wave equation. Therefore, it remains exact for solutions of the two-dimensional wave equation, which depend only on $x$ and $t$ and thus impinge on the artificial boundary with a normal angle of incidence. Next, we include one additional term of the Taylor expansion in the approximation, $\sqrt{1-s^{2}} \simeq 1-\frac{s^{2}}{2}$. This yields the second-order Engquist-Majda boundary condition

$$
\frac{\partial \widehat{u}}{\partial x} \simeq-\mathrm{i} \omega\left(1-\frac{1}{2}\left(\frac{\xi}{\omega}\right)^{2}\right) \widehat{u} \Rightarrow\left(\frac{\partial^{2}}{\partial t^{2}}+\frac{\partial^{2}}{\partial x \partial t}-\frac{1}{2} \frac{\partial^{2}}{\partial y^{2}}\right) u=0, \quad x=L .
$$

Equation (2.12) remains exact at normal incidence, since we can rewrite it in the equivalent form as

$$
\left(\partial_{t}+\partial_{x}\right)\left(\partial_{t}+\partial_{x}\right) u=0, \quad x=L,
$$

by using (2.8). The inclusion of even higher order terms of the Taylor expansion to improve the accuracy of the approximation ceases to yield well-posed boundary conditions. Although 
this difficulty can be overcome by the use of rational (Padé) approximations, the high-order derivatives involved in these boundary conditions greatly complicate their use in any numerical scheme. As a result, the first- and second-order boundary conditions are most commonly used in practice.

Various other (e.g., Chebyshev) approximations of $\sqrt{1-s^{2}}$ were proposed to design improved local boundary conditions. Eventually, Higdon [15] showed that all these boundary conditions are particular cases of the following general class of boundary operators

$$
\left(\cos \alpha_{p} \frac{\partial}{\partial t}+\frac{\partial}{\partial x}\right) \cdots\left(\cos \alpha_{1} \frac{\partial}{\partial t}+\frac{\partial}{\partial x}\right) u=0, \quad x=L,
$$

where $\alpha_{1}, \cdots, \alpha_{p}$ are arbitrary parameters. For instance, the second-order Engquist-Majda boundary condition (2.13) results from setting $\alpha_{1}=0^{\circ}$ and $\alpha_{2}=0^{\circ}$ in (2.14). This general formulation, written as the product of first-order differential operators $\left(\cos \alpha_{i} \partial_{t}+\partial_{x}\right)$, provides a new, more intuitive, interpretation for the effectiveness of absorbing boundary conditions. Since any such differential operator perfectly annihilates plane waves with angle of incidence $\pm \alpha_{i}$, the artificial boundary will appear absolutely transparent at the discrete angles of incidence $\alpha_{1}, \cdots, \alpha_{p}$. The choice of $\alpha_{1}, \cdots, \alpha_{p}$ is arbitrary and can be adapted to any given situation.

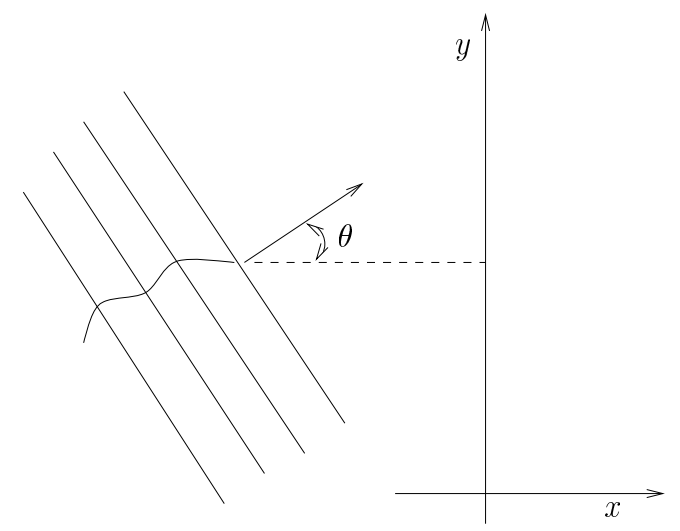

Figure 3 A traveling plane wave with an angle of incidence $\theta$.

Nevertheless, all absorbing boundary conditions remain only approximations to the exact boundary condition (2.11); therefore, they generate some spurious reflection at $x=L$. How large is the amount of reflection for a specific boundary condition? Recall that any solution of the (homogeneous) wave equation can be represented by the superposition of plane waves. In Figure 3 we observe a plane wave, which impinges on the artificial boundary at $x=L$ with an angle of incidence $\theta$. The linearity of both the wave equation (2.8) and the boundary condition (2.14) imply that any reflected wave necessarily propagates with the same frequency as the incident wave. Hence the solution consists of an outgoing wave, whose amplitude we normalize to one, and an incoming spurious wave with amplitude $|r|$ :

$$
u(x, y, t)=\mathrm{e}^{\mathrm{i}(k x+\ell y-\omega t)}+r \mathrm{e}^{\mathrm{i}(-k x+\ell y-\omega t)}, \quad k, \omega \geq 0,
$$

where $r=r\left(\theta ; \alpha_{1}, \cdots, \alpha_{p}\right)$ depends on the angle of incidence $\theta$, defined by $\tan \theta=\frac{\ell}{k}$, and the fixed parameters $\alpha_{1}, \cdots, \alpha_{p}$. In Figure 4 we compare the effectiveness of three absorbing 
boundary conditions by displaying the amount of reflection $|r|$ versus the angle of incidence $\theta$. The choice $\alpha_{1}=0^{\circ}$ corresponds to the first, whereas $\alpha_{1}=0^{\circ}$ and $\alpha_{2}=0^{\circ}$ correspond to the second Engquist-Majda boundary condition. Alternatively, the popular parameter choice $\alpha_{1}=0^{\circ}$ and $\alpha_{2}=60^{\circ}$ annihilates incoming waves at normal incidence and at $60^{\circ}$ angle of incidence. All other angles of incidence will generate some spurious reflection, which is very small close to normal incidence but rapidly increases as grazing incidence is approached.

Reflection coefficient [\%]

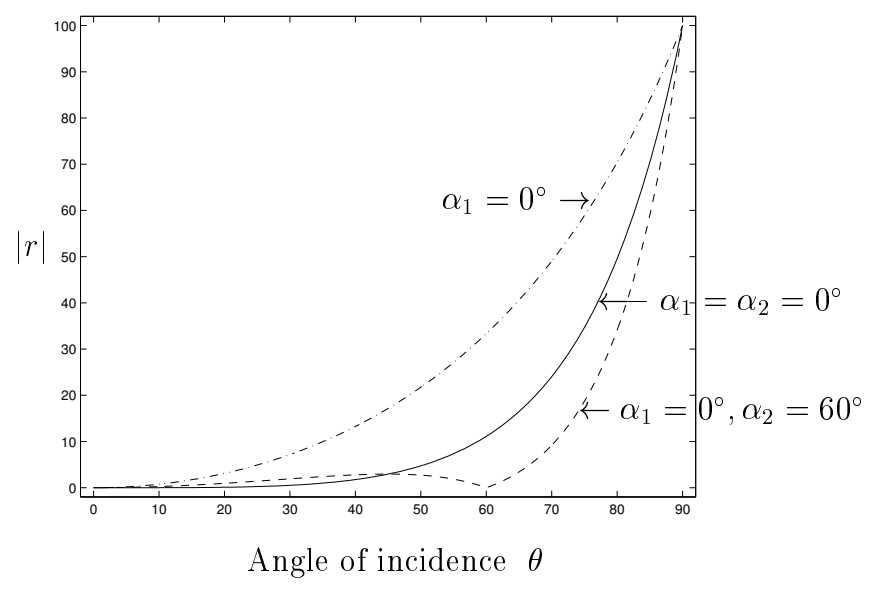

Figure 4 Amount of spurious reflection (in percent) caused by the use of the boundary condition (2.14) for a plane wave with angle of incidence $\theta$.

\subsection{High-order local nonreflecting boundary conditions}

The local absorbing boundary conditions described in the previous section can be made arbitrarily accurate, but in practice the resulting increasingly higher order derivatives greatly complicate their use in any numerical scheme. As a result, the first- and second-order boundary conditions are most commonly used in practice. If even higher accuracy is needed, the artificial boundary then needs to be moved farther away from the scatterer. Hence the absorbing boundary conditions from Section 2 do not fully satisfy the demand for increasingly accurate and efficient modern numerical methods to solve complex time-dependent scattering problems in unbounded domains.

Starting from a convergent series representation of the scattered field in inverse powers of distance, Hagstrom and Hariharan [9] derived a nonreflecting boundary condition of arbitrarily high order, in the special case when $B$ is a sphere. Thus, let $B$ be the sphere of radius $R$ and assume that $u$ satisfies the homogeneous wave equation

$$
\frac{\partial^{2} u}{\partial t^{2}}-c^{2} \Delta u=0
$$

with zero initial condition outside $B$. Starting from the convergent expansion

$$
u(r, \theta, \phi, t)=\sum_{j=1}^{\infty} \frac{f_{j}(t-r, \theta, \phi)}{r^{j}}, \quad r>R,
$$


where $r, \theta$ and $\phi$ are spherical coordinates, Hagstrom and Hariharan [9] derived the following exact local NRBC:

$$
\begin{aligned}
& \left(\frac{1}{c} \frac{\partial}{\partial t}+\frac{\partial}{\partial r}+\frac{1}{r}\right) u=w_{1} \\
& \left(\frac{1}{c} \frac{\partial}{\partial t}+\frac{k}{r}\right) w_{k}=\frac{1}{4 R^{2}}\left(k(k-1)+\Delta_{S}\right) w_{k-1}+w_{k+1}
\end{aligned}
$$

for $k=1,2, \cdots$, and $w_{0}=2 u$. Here, $\Delta_{S}$ denotes the Laplace-Beltrami operator in spherical coordinates $(r, \theta, \phi)$,

$$
\Delta_{S}=\frac{1}{\sin \theta} \frac{\partial}{\partial \theta}\left(\sin \theta \frac{\partial}{\partial \theta}\right)+\frac{1}{\sin ^{2} \theta} \frac{\partial^{2}}{\partial \phi^{2}} .
$$

In fact in 1980, Bayliss and Turkel [17] started from that same infinite series representation and derived a hierarchy of local absorbing boundary conditions in spherical coordinates. Similar to the boundary conditions of Engquist and Majda [3,4], it also requires increasingly higher order derivatives for improved accuracy.

The boundary condition (2.18) is local in space and time yet does not involve high-order derivatives, but instead an infinite sequence of auxiliary variables $w_{k}$ defined on $B$. In practice, only a finite number of auxiliary functions $w_{k}, k=1, \cdots, P$ is used setting $w_{P+1}=0$. Then, in general the boundary condition is no longer exact, but it remains exact for solutions which consist of a finite combination of vector spherical harmonics up to order $P$. Imposition of the boundary condition at any fixed radius $R$ thus yields at least spectral accuracy for smooth wave fields with increasing $P$. Therefore (2.18) is exact in the same sense as the conditions proposed in $[7,8,16]$, namely that $P$ can always be chosen sufficiently large so that the error introduced at $\mathcal{B}$ is smaller than the discretization error inside $\Omega$, without moving $\mathcal{B}$ farther away from the scatterer. However, this new boundary condition does not require any spherical harmonics or inner products with them; hence, it is somewhat easier and cheaper to implement.

By combining ideas from [9] and [16], the above approach was recently extended to Maxwell's equations in three space dimensions (see [11]). Again, outside $B$, the medium is assumed to be linear, homogeneous, isotropic, of constant electric permittivity $\varepsilon$, of constant magnetic permeability $\mu$, and of zero conductivity. In addition, we assume that at $t=0$ the scattered field is confined to the computational domain inside $B$. Then, the following exact nonreflecting boundary condition holds (see [11]):

$$
\begin{aligned}
& \widehat{\boldsymbol{r}} \times \operatorname{curl} \boldsymbol{E}-\frac{1}{c} \frac{\partial \boldsymbol{E}^{\tan }}{\partial t}=\boldsymbol{w}^{1}, \\
& \frac{1}{c} \frac{\partial \boldsymbol{w}^{1}}{\partial t}+\frac{\boldsymbol{w}^{1}}{r}=\frac{1}{2 r^{2}}\left[\overrightarrow{\operatorname{curl}}_{S} \operatorname{curl}_{S} \boldsymbol{E}+\sqrt{\frac{\mu}{\varepsilon}} \widehat{\boldsymbol{r}} \times \overrightarrow{\operatorname{curl}}_{S} \operatorname{curl}_{S} \boldsymbol{H}\right]+\boldsymbol{w}^{2}, \\
& \frac{1}{c} \frac{\partial \boldsymbol{w}^{p}}{\partial t}+\frac{p}{r} \boldsymbol{w}^{p}=\frac{1}{4 r^{2}}\left(\vec{\Delta}_{S}+p(p-1)\right) \boldsymbol{w}^{p-1}+\boldsymbol{w}^{p+1}, \quad p \geq 2 .
\end{aligned}
$$

Again, the boundary condition (2.20)-(2.22) is local both in space and time. It only involves first time derivatives and second tangential derivatives of $\boldsymbol{E}$ and of the (unknown) auxiliary functions $\boldsymbol{w}^{p}, p \geq 1$, which satisfy (2.21)-(2.22). Since at least two scalar potentials are necessary to represent the general three-dimensional electro-magnetic field in free space, this 
boundary condition is optimal in the sense that the number of auxiliary variables required is minimal.

\section{Multiple Scattering Problems}

When the scatterer consists of several obstacles, which are well separated from each other, the use of a single artificial boundary to enclose the entire scattering region becomes too expensive. Instead, it is preferable to enclose every sub-scatterer by a separate artificial boundary $B_{i}$. Then we seek an exact boundary condition on $B=\cup B_{i}$, where each $B_{i}$ surrounds a single computational sub-domain $\Omega_{i}$. This boundary condition must not only let outgoing waves leave $\Omega_{i}$ without spurious reflection from $B_{i}$, but also propagate the outgoing wave from $\Omega_{i}$ to all other sub-domains, which it may reenter subsequently. To derive such an exact boundary condition, an analytic representation of the solution everywhere in the exterior region is needed.

\subsection{The one-dimensional case}

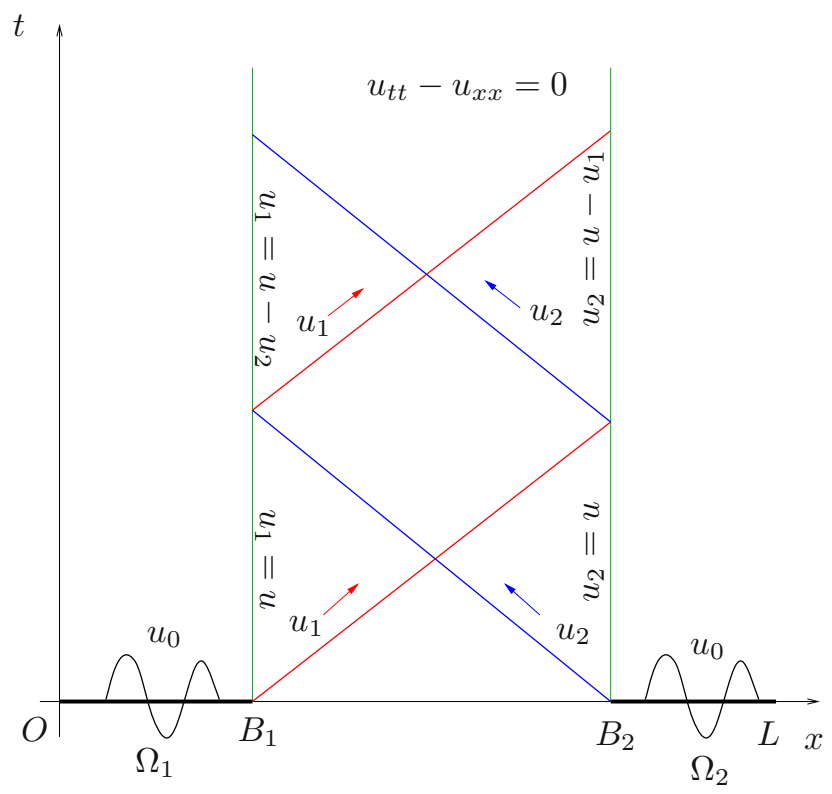

Figure 5 Multiple scattering in one space dimension.

To illustrate the basic principle underlying the NRBC for multiple scattering problems, we first consider the following simple one-dimensional Cauchy problem:

$$
\begin{aligned}
& \frac{\partial^{2} u}{\partial t^{2}}-\frac{\partial^{2} u}{\partial x^{2}}=f(x, t), \quad-\infty<x<\infty, t>0, \\
& u(x, 0)=u_{0}(x) \\
& u_{t}(x, 0)=v_{0}(x) .
\end{aligned}
$$

We assume that the initial disturbance and the forcing are supported inside the region $\Omega=$ $\Omega_{1} \cap \Omega_{2}$, with $\Omega_{1}=\left[0, B_{1}\right]$ and $\Omega_{2}=\left[B_{2}, L\right], 0<B_{1}<B_{2}<L$, that is $\operatorname{supp}\left\{u_{0}, v_{0}, f(\cdot, t)\right\} \subset \Omega$ 
(see Figure 5). We now wish to restrict the computation to the sub-region $\Omega$; therefore we need to impose appropriate boundary conditions at $x=0, B_{1}, B_{2}$ and $L$ to ensure that the solution in $\Omega$ coincides with the solution $u$ of the original Cauchy problem for all time. Because $u$ is purely outgoing for $x<0$ and $x>L$, the NBC at $x=0$ and $x=L$ correspond to the standard artificial boundary conditions for single scattering (see Subsection 2.1), that is,

$$
\begin{aligned}
& \left(\frac{\partial}{\partial x}-\frac{\partial}{\partial t}\right) u=0, \quad x=0, \\
& \left(\frac{\partial}{\partial x}+\frac{\partial}{\partial t}\right) u=0, \quad x=L,
\end{aligned}
$$

which require no further discussion. We now focus on the two remaining artificial boundary points at $x=B_{1}$ and $x=B_{2}$, where $u$ is not purely outgoing. Because $u$ satisfies the homogeneous wave equation in $\left[B_{1}, B_{2}\right]$, it is the superposition of a left and right moving wave there, that is,

$$
u(x, t)=u_{1}(x, t)+u_{2}(x, t)
$$

with

$$
u_{1}(x, t)=f(x-t), \quad u_{2}(x, t)=g(x+t) .
$$

Moreover, if we require that $\operatorname{supp}\left\{u_{1}\right\} \subset \Omega_{1}$ and $\operatorname{supp}\left\{u_{2}\right\} \subset \Omega_{2}$ at $t=0, u_{1}$ and $u_{2}$ are uniquely defined for all time (see [18]). At $x=B_{1}$, an exact NRBC is

$$
\left(\frac{\partial}{\partial x}+\frac{\partial}{\partial t}\right) u=\left(\frac{\partial}{\partial x}+\frac{\partial}{\partial t}\right) u_{1}+\left(\frac{\partial}{\partial x}+\frac{\partial}{\partial t}\right) u_{2}=\left(\frac{\partial}{\partial x}+\frac{\partial}{\partial t}\right) u_{2},
$$

since $u_{1}$ is outgoing here.

Thus to impose the exact NRBC at $x=B_{1}$, we must be able to evaluate $u_{2}$ there. Here we need to distinguish initial times up to $t=B_{2}-B_{1}$ from later times $t \geq B_{2}-B_{1}$ :

(1) $0 \leq t<B_{2}-B_{1}$. Due to the finite propagation speed (here equal to one), $u_{2}$ has not reached $\Omega_{1}$ yet; hence, it is still zero at $x=B_{1}$ and (3.4) reduces to the standard NRBC for purely outgoing solutions.

(2) $B_{2}-B_{1} \leq t . u_{2}$ no longer vanishes at $x=B_{1}$, however it is fully determined by its past values at $x=B_{2}$ through

$$
u_{2}\left(B_{1}, t\right)=u_{2}\left(B_{2}, t-\left(B_{2}-B_{1}\right)\right) .
$$

How do we determine $u_{2}$ at $x=B_{2}$ ? Recall that we are only computing $u$ (and not $u_{1}$ or $u_{2}$ ) inside $\Omega$. Again, during initial times $t<B_{2}-B_{1}$, we have $u_{2}=u$ at $x=B_{2}$. To determine $u_{2}$ at later times, we recall that

$$
u(x, t)=u_{1}(x, t)+u_{2}(x, t), \quad \forall x \in\left[B_{1}, B_{2}\right], t>0 .
$$

Therefore we obtain $u_{2}$ at $x=B_{2}$ by subtracting from $u$ the value of $u_{1}$ there, which again is determined by its past values on $B_{1}$, that is,

$$
u_{2}\left(B_{2}, t\right)=u\left(B_{2}, t\right)-u_{1}\left(B_{2}, t\right)=u\left(B_{2}, t\right)-u_{1}\left(B_{1}, t-\left(B_{2}-B_{1}\right)\right) .
$$

Hence in every time step of the numerical scheme, we concurrently update the new values of $u_{1}$ and $u_{2}$ at $x=B_{1}, B_{2}$ respectively. This requires the additional storage of past values $u_{i}$ at $x=B_{i}, i=1,2$, for the finite time window $\left[t-\left(B_{2}-B_{1}\right), t\right]$. 


\subsection{The three-dimensional case}

For simplicity, we consider a scattering problem with two bounded disjoint scatterers, each surrounded by a sphere $B_{i}$ of radius $R_{i}, i=1,2$. Hence, the entire artificial boundary $B=$ $B_{1} \cup B_{2}$ and the computational domain $\Omega=\Omega_{1} \cup \Omega_{2}$. In contrast to the situation of single scattering in Section 2, we cannot simply expand $u$ outside $B$ as a superposition of purely outgoing wave fields. In fact, since part of the scattered field leaving $\Omega_{1}$ will reenter $\Omega_{2}$ at later times, and vice versa, $u$ is not outgoing outside $\Omega$. Thus, the boundary condition we seek at $B$ must not only let outgoing waves leave $\Omega_{1}$ without spurious reflection from $B_{1}$, but also propagate those waves to $\Omega_{2}$, and so forth, without introducing any spurious reflections.

Following [18], we first decompose the scattered field $u$ in two wave fields $u=u_{1}+u_{2}$, where $u_{i}$ is purely outgoing as seen from $\Omega_{i}$. The two wave fields $u_{1}$ and $u_{2}$ both solve the homogeneous wave equation (2.16) outside $\Omega$, and their sum coincides with $u$. The outgoing field $u_{1}^{\text {out }}$, as seen from $\Omega_{1}$, is fully determined by its boundary values on $B_{1}$, while the incoming field $u_{12}^{\mathrm{in}}$ is fully determined by its boundary values on $B_{2}$. Hence

$$
\begin{aligned}
& u_{1}^{\text {out }}+u_{12}^{\text {in }}=\left.u\right|_{B_{1}}, \\
& u_{2}^{\text {out }}+u_{21}^{\text {in }}=\left.u\right|_{B_{2}},
\end{aligned}
$$

where $u_{i}^{\text {out }}$ is the outgoing wave field from $\Omega_{i}$ and $u_{i j}^{\text {in }}$ is the incoming wave propagating from $\Omega_{j}$ to $\Omega_{i}$.

Next, we apply $c^{-1} \partial_{t}+\partial_{r_{i}}+r_{i}^{-1}$ in local spherical coordinates $\left(r_{i}, \theta_{i}, \phi_{i}\right)$ to $u$ on each artificial boundary component $B_{i}, i=1,2$. This yields the following exact local NBC for multiple scattering (see [19]):

$$
\begin{aligned}
& \left.\mathcal{B}_{1} u\right|_{B_{1}}=\left.\left(\frac{1}{c} \frac{\partial}{\partial t}+\frac{\partial}{\partial r_{1}}+\frac{1}{R_{1}}\right) u\right|_{B_{1}}=\mathcal{B}_{1} u_{1}^{\text {out }}+\mathcal{B}_{1} u_{12}^{\text {in }}, \quad \text { on } B_{1}, \\
& \left.\mathcal{B}_{2} u\right|_{B_{2}}=\left.\left(\frac{1}{c} \frac{\partial}{\partial t}+\frac{\partial}{\partial r_{2}}+\frac{1}{R_{2}}\right) u\right|_{B_{2}}=\mathcal{B}_{2} u_{2}^{\text {out }}+\mathcal{B}_{2} u_{21}^{\text {in }}, \quad \text { on } B_{2} .
\end{aligned}
$$

To evaluate $\mathcal{B}_{1} u_{1}^{\text {out }}$ we use $(2.18)$ at $B_{1}$, whereas to evaluate $\mathcal{B}_{1} u_{12}^{\text {in }}$ we use past values for $u_{2}$ and the corresponding auxiliary functions on $B_{1}$. The needed past values of $w_{k}$ are stored on each $B_{i}$ at regular time and angular intervals and calculated, as needed, via local spline interpolation (see [19]). Because those values are time-retarded, they are already known, so that the entire scheme remains explicit in time. Remarkably, the information transfer (of time retarded values) between sub-domains occurs only across those parts of the artificial boundary, where outgoing rays intersect neighboring sub-domains, i.e., typically only across a fraction of the artificial boundary.

\section{Numerical Experiment}

We shall now illustrate the accuracy of local nonreflecting boundary conditions via the following numerical experiment. Consider a spherical inclusion of radius $r_{0}>0$ located inside an unbounded inhomogeneous acoustic medium. At the sound-soft interface of the inclusion we impose a time-dependent pressure field which corresponds to an outgoing spherical wave field, 
initiated by an off-centered Gaussian point source. Located on the $z$-axis at distance $d<r_{0}$ from the origin, its time dependence is shown in Figure 6. Hence at the surface of the cavity, $r=r_{0}$, the imposed time-dependent acoustic field is determined by

$$
u\left(r_{0}, \theta, t\right)=\frac{1}{r_{d}} \exp \left(-\frac{\left(r_{d}-c_{\min } t+0.2\right)^{2}}{\sigma^{2}}\right),
$$

where $r_{d}=\sqrt{r_{0}^{2}+d^{2}-2 r_{0} d \cos (\theta)}, \sigma=\frac{0.25}{\sqrt{-\log \alpha}}, \alpha=10^{-7}$, and $c_{\min }=0.5$. Here, $r_{d}$ corresponds to the distance of any point $\left(r_{0}, \theta\right)$ from the point source at distance $d$ from the origin.

The sound speed in the surrounding medium varies from $c_{\min }$ to $c_{\max }$ as a function of distance only. For $r \geq 1$, the velocity profile shown in Figure 6 is constant and equal to $c_{\max }$ normalized to one. Hence the inhomogeneous surrounding medium, initially at rest, is expected to act as a spherical wave guide around the cavity. The unbounded exterior is now truncated at $R=1$, where we apply the high-order local conditions (2.18) with varying $P$, noting that with $P=0$ the boundary condition (2.18) corresponds to the first-order Engquist-Majda condition in spherical coordinates.
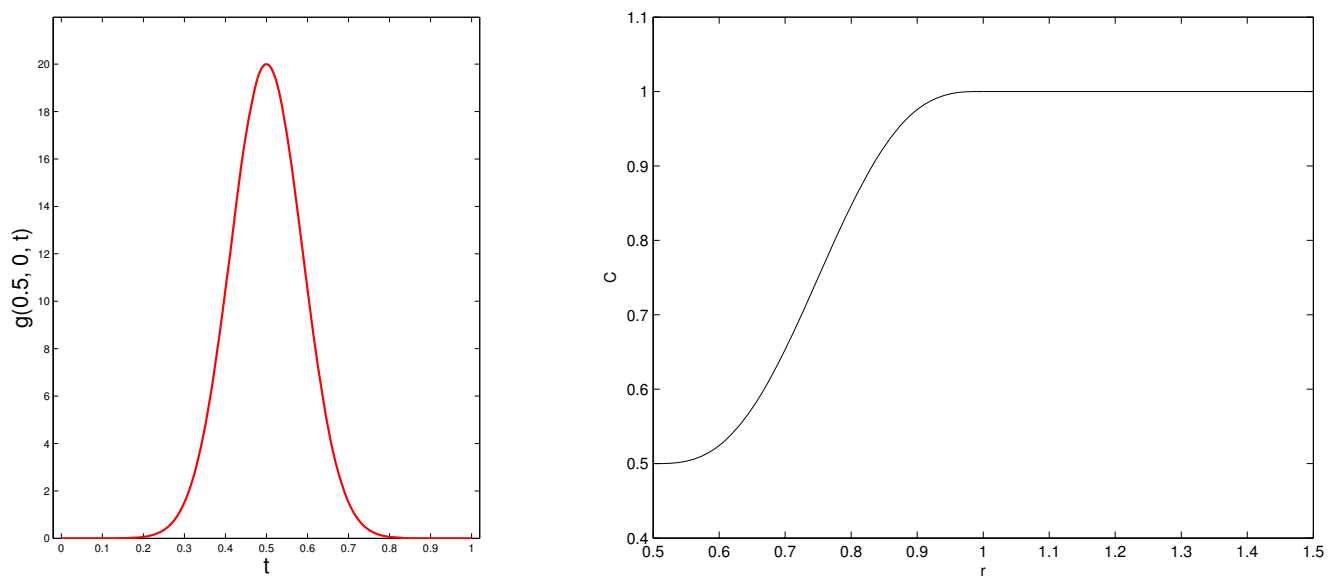

Figure 6 Left: the time dependence of the Gaussian point source. Right: the velocity profile $c(r)$.

Although this test problem is three-dimensional, it is axisymmetric about the $z$-axis, that is, the solution is independent of $\phi$, so that we can restrict the computations to the two-dimensional region $\Omega$, determined by $r_{0} \leq r \leq R, 0 \leq \theta \leq \pi$. Inside $\Omega$ we use the standard second-order centered finite differences on an $80 \times 480$ polar equidistant mesh, combined with the explicit second-order leap-frog scheme in time. At the artificial boundary $B$ located at $r=R$, the boundary condition (2.18) is discretized in space using centered second-order finite differences and in time as described in [9].

Since no simple analytical expression for the exact solution is available here, we shall compute a reference solution in a much larger domain. Due to the finite speed of propagation, any spurious reflection will then be postponed to later times and thus not affect the reference solution inside $\Omega$ until $T=7.5$. In Figure 7 we observe how the spherical wave front penetrates the 

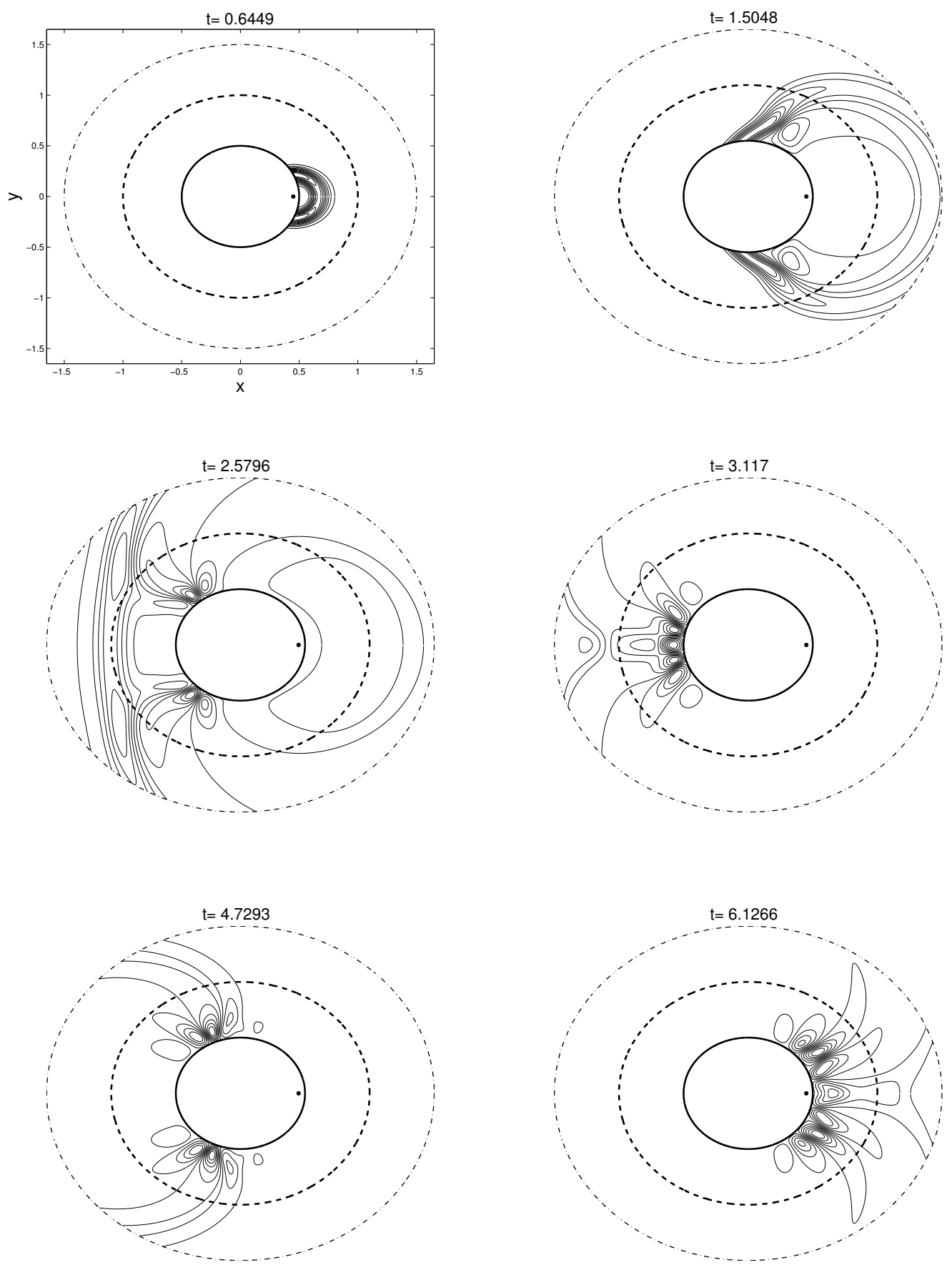

Figure 7 Scattering from a spherical wave guide: snapshots of the reference solution at different times. The three circles drawn are located at $r=0.5,1,1.5$. The Gaussian point source is located outside the computational domain at $r=0.45, \theta=0$. 
acoustic medium to the right of the cavity and then progresses around it, noting the distorted wave front due to the varying velocity profile around $t=1.5$. By $t=3$ the main wave front has left the computational domain, yet part of the wave energy remains trapped inside the wave guide and continues to travel around the cavity.

We now compare the exact (numerical reference) solution with that obtained by imposing the boundary condition (2.18) for varying $P$ at the fixed location $r=0.75, \theta=\frac{3 \pi}{4}$, located well inside $\Omega$. In Figure 8 the numerical solutions obtained with $P=0, P=1$ and $P=5$ are shown. The numerical solution obtained with $P=0$ strongly deviates from the exact solution past $t=2$. We recall that the error observed here is solely due to the approximate nature of the boundary condition and thus cannot be improved upon by refining the mesh. The solution obtained with $P=1$ clearly displays a significant improvement in accuracy. Nonetheless, we find again deviations of $5-10 \%$ around $t=3.5$, for instance. As we further increase $P$, those spurious reflections essentially disappear and cannot be observed anymore at this scale. Hence their amplitude now lies below the discretization error. Further mesh refinement in the interior, however, would generally require further increase in $P$, as both error components need to be reduced systematically to achieve convergence.

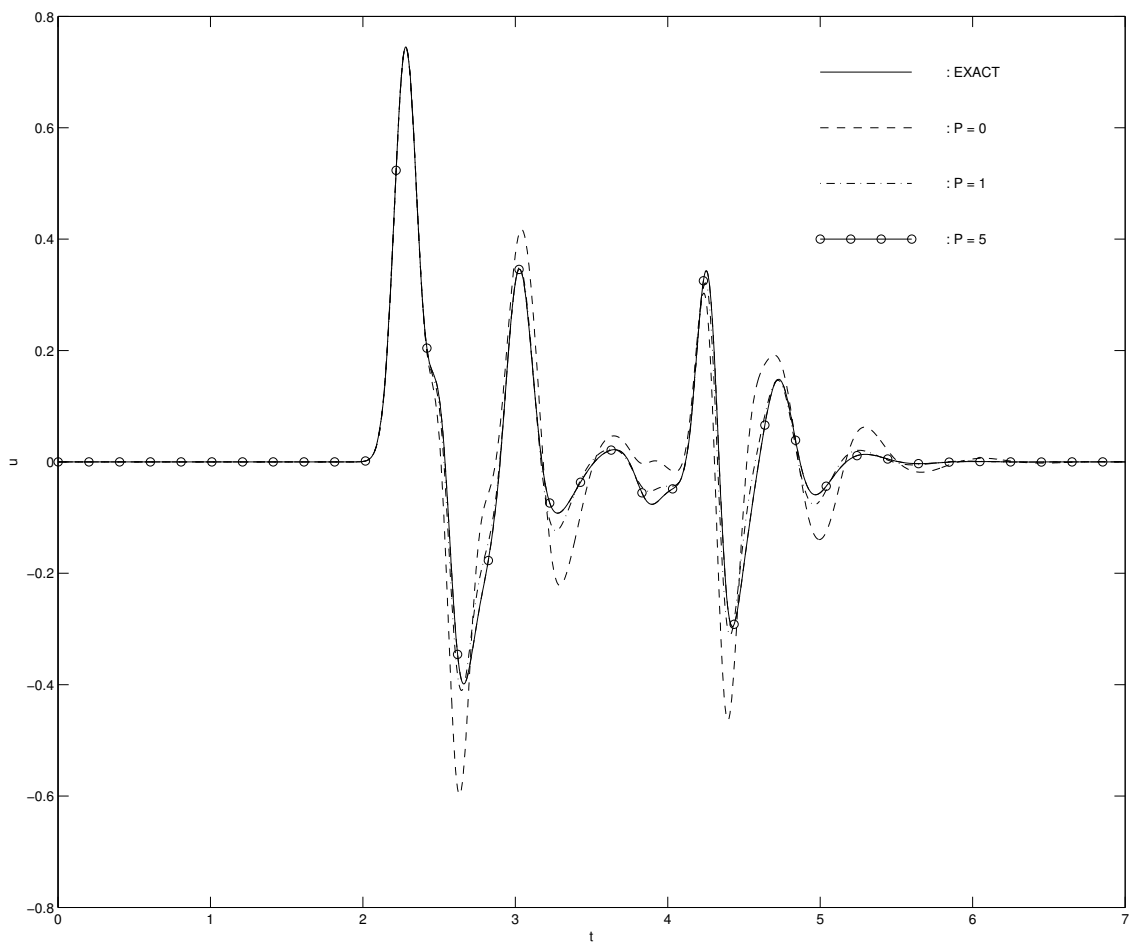

Figure 8 The numerical solutions computed using the boundary conditions (2.18) with $P=0, P=1$ and $P=5$, are compared with the exact solution at $r=0.75, \theta=\frac{3 \pi}{4}$. 


\section{Conclusion}

The constant demand for increasingly accurate, efficient, and robust numerical methods, which can handle a wide variety of physical phenomena, spurs the search for improvements in absorbing boundary conditions. The frustration is all too obvious, when the gains made in the interior by using sophisticated numerical methods, such as high order and adaptive methods, are annihilated at the artificial boundary by the use of an inaccurate boundary condition.

Among many different approaches nowadays available to truncate the unbounded exterior and achieve convergence at a reasonable computational cost, local absorbing boundary conditions remain probably the simplest and most flexible approach. Because they are completely local, they apply to all (convex) artificial boundaries and require no special functions or damping parameters in the exterior. Moreover, they are easily coupled with standard finite difference or finite element methods in the interior and have been found very accurate in practical computations. In contrast to the popular perfectly matched layer approach, high-order local nonreflecting boundary conditions can also be extended to multiple scattering problems, as they yield an efficient analytical representation of the solution everywhere outside the computational domain.

\section{References}

[1] Givoli, D., Numerical Methods for Problems in Infinite Domains, Studies in Applied Mechanics, Vol. 33, Elsevier, Amsterdam, 1992.

[2] Smith, W. D., Nonreflecting plane boundary for wave propagation problems, J. Comput. Phys., 15, 1974, 492-503.

[3] Engquist, B. and Majda, A., Absorbing boundary conditions for the numerical simulation of waves, Math. Comput., 31, 1977, 629-651.

[4] Engquist, B. and Majda, A., Absorbing boundary conditions for numerical simulation of waves, Proc. Natl. Acad. Sci. USA, 74, 1977, 1765-1766.

[5] Bérenger, J.-P., A perfectly matched layer for the absorbtion of electromagnetic waves, J. Comput. Phys., 114, 1994, 185-200.

[6] Ergin, A. A., Shanker, B. and Michielssen, E., Fast evaluation of three-dimensional transient wave fields using diagonal translation operators, J. Comput. Phys., 146, 1998, 157-180.

[7] Grote, M. J. and Keller, J. B., Exact nonreflecting boundary conditions for the time dependent wave equation, SIAM J. Appl. Math., 55, 1995, 280-297.

[8] Grote, M. J. and Keller, J. B., Nonreflecting boundary conditions for time-dependent scattering, J. Comput. Phys., 127, 1996, 52-65.

[9] Hagstrom, T. and Hariharan, S. I., A formulation of asymptotic and exact boundary conditions using local operators, Appl. Numer. Math., 27, 1998, 403-416.

[10] Hagstrom, T. and Warburton, T., A new auxiliary variable formulation of high-order local radiation conditions: corner compatibility conditions and extensions to first-order systems, Wave Motion, 39, 2004, 327-338.

[11] Grote, M. J., Local nonreflecting boudnary condition for Maxwell's equations, Comp. Methods Appl. Mech. Eng., 195, 2006, 3691-3708.

[12] Hagstrom, T., Radiation boundary conditions for the numerical simulation of waves, Acta Numer., 8, 1999, $47-106$.

[13] Tsynkov, S. V., Numerical solution of problems on unbounded domains: a review, Appl. Numer. Math., 27, 1998, 465-532.

[14] Givoli, D., High-order local non-reflecting boundary conditions: a review, Wave Motion, 39, 2004, 319-326. 
[15] Higdon, R. L., Numerical absorbing boundary conditions for the wave equation, Math. Comput., 49, 1987, 65-90.

[16] Grote, M. J. and Keller, J. B., Nonreflecting boundary conditions for Maxwell's equations, J. Comput. Phys., 139, 1998, 327-342.

[17] Bayliss, A. and Turkel, E., Radiation boundary conditions for wave-like equations, Comm. Pure Appl. Math., 33, 1980, 707-725.

[18] Grote, M. J. and Kirsch, C., Nonreflecting boundary condition for time-dependent multiple scattering, $J$. Comput. Phys., 221, 2007, 41-62.

[19] Grote, M. J. and Sim, I., Local nonreflecting boundary conditions for time-dependent multiple scattering, in preparation. 